\title{
Plasmapheresis for Human Source Plasma
}

National Cancer Institute

\section{Source}

National Cancer Institute. Plasmapheresis for Human Source Plasma. NCI Thesaurus.

Code C113043.

Collection of the fluid portion of human blood for use in blood products by plasmapheresis. 